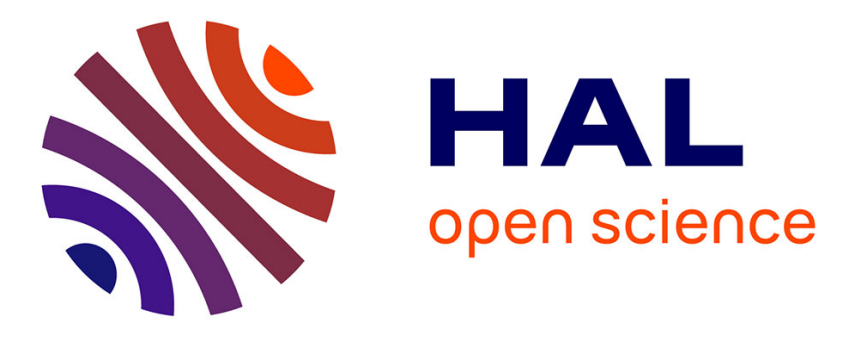

\title{
Euler polynomials and identities for non-commutative operators
}

\author{
Valerio de Angelis, Christophe Vignat
}

\section{To cite this version:}

Valerio de Angelis, Christophe Vignat. Euler polynomials and identities for non-commutative operators. Journal of Mathematical Physics, 2015, 10.1063/1.4938077 . hal-01248828

\section{HAL Id: hal-01248828 \\ https://hal.science/hal-01248828}

Submitted on 10 Apr 2020

HAL is a multi-disciplinary open access archive for the deposit and dissemination of scientific research documents, whether they are published or not. The documents may come from teaching and research institutions in France or abroad, or from public or private research centers.
L'archive ouverte pluridisciplinaire HAL, est destinée au dépôt et à la diffusion de documents scientifiques de niveau recherche, publiés ou non, émanant des établissements d'enseignement et de recherche français ou étrangers, des laboratoires publics ou privés. 


\title{
Euler Polynomials and Identities for Non-Commutative Operators
}

\author{
Valerio De Angelis and Christophe Vignat \\ Department of Mathematics, Xavier University of Louisiana, New Orleans, \\ Department of Mathematics, Tulane University, New Orleans, \\ L.S.S. Supelec Université Paris Sud Orsay
}

\begin{abstract}
Three kinds of identities involving non-commutating operators and Euler and Bernoulli polynomials are studied. The first identity, as given in [2] by Bender and Bettencourt, expresses the nested commutator of the Hamiltonian and momentum operators as the commutator of the momentum and the shifted Euler polynomial of the Hamiltonian. The second one, due to J.-C. Pain [3], links the commutators and anti-commutators of the monomials of the position and momentum operators. The third appears in a work by Figuieira de Morisson and Fring [9] in the context of non-Hermitian Hamiltonian systems. In each case, we provide several proofs and extensions of these identities that highlight the role of Euler and Bernoulli polynomials.
\end{abstract}




\section{INTRODUCTION}

Special functions appear as a natural tool in many areas of theoretical physics; in quantum physics, Hermite polynomials are the natural basis to describe the behavior of the quantum harmonic oscillator. The Euler and Bernoulli polynomials are ubiquitous in number theory and combinatorics; in physics, they appear in various ways, such as in their association with zeta functions and in their rich interplay with particle physics; see [7] for examples such as the Casimir effect and string theory.

Bernoulli and Euler polynomials also appear in the field of non-commutative operators in quantum physics, in more subtle ways; for example, the Bernoulli numbers appear in the the linear term of the celebrated Baker-Campbell-Hausdorff formula. Their role in quantum algebras is also detailed in [10]. This paper describes three distinct contexts of quantum physics in which the Bernoulli and Euler polynomials play an important role.

Define the commutator of two operators $p$ and $q$ as

$$
[p, q]=p q-q p
$$

and their anti-commutator as

$$
\{p, q\}=p q+q p
$$

Define moreover the nested (or iterated) commutators

$$
[p, q]_{2}=[[p, q], q],[p, q]_{3}=[[[p, q], q], q]=\left[[p, q]_{2}, q\right]
$$

and more generally

$$
[p, q]_{n}=\left[[p, q]_{n-1}, q\right], n \geq 1
$$

and accordingly the nested anti-commutators as

$$
\{p, q\}_{2}=\{\{p, q\}, q\},\{p, q\}_{3}=\{\{\{p, q\}, q\}, q\}=\left\{\{p, q\}_{2}, q\right\}
$$

and more generally

$$
\{p, q\}_{n}=\left\{\{p, q\}_{n-1}, q\right\}, n \geq 1
$$

For example

$$
\{p, q\}_{2}=\{p q+q p, q\}=p q^{2}+2 q p q+q^{2} p
$$

and

$$
[p, q]_{2}=[p q-q p, q]=p q^{2}-2 q p q+q^{2} p .
$$

It can be checked by induction on $n$ that the general cases are

$$
[p, q]_{n}=\sum_{k=0}^{n}\left(\begin{array}{l}
n \\
k
\end{array}\right)(-1)^{k} q^{k} p q^{n-k}
$$

and

$$
\{p, q\}_{n}=\sum_{k=0}^{n}\left(\begin{array}{l}
n \\
k
\end{array}\right) q^{k} p q^{n-k}
$$

as a consequence, the exponential generating functions of these two sequences are

$$
\sum_{n \geq 0}[p, q]_{n} \frac{z^{n}}{n !}=e^{-z q} p e^{z q}, \sum_{n \geq 0}\{p, q\}_{n} \frac{z^{n}}{n !}=e^{z q} p e^{z q}
$$


Note that $[p, q]=[p, q]_{1}$ and $\{p, q\}=\{p, q\}_{1}$.

In the sequel, we will consider the more particular case where the non commutative operators $p$ and $q$ satisfy the identity

$$
[q, p]=q p-p q=\imath
$$

where $\imath^{2}=-1$. A more rigorous notation would be

$$
q p-p q=\imath I
$$

where $I$ is the identity operator, but in the absence of ambiguity, we will systematically use the simplified notation (I.1). For example, take $q=\imath \frac{d}{d x}$ and $p=x$ in the sense that $q$ and $p$ act on any differentiable function $f$ as follows

$$
q f(x)=\imath \frac{d}{d x} f(x)=\imath f^{\prime}(x)
$$

and

$$
p f(x)=x f(x) .
$$

Then $(q p-p q) f=\imath f$ so that indeed

$$
q p-p q=\imath,
$$

in the sense that, for any differentiable function $f$,

$$
(q p-p q) f=\imath f .
$$

However, we wish to prove the results stated in the next sections in the general case of two arbitrary operators $p$ and $q$, assuming only that $p$ and $q$ satisfy (I.1).

Let us introduce moreover another operator, the Hamiltonian operator $H$, as

$$
H=\frac{1}{2}\left(p^{2}+q^{2}\right) .
$$

Our aim in this paper is to study three types of identities on specific commutators and anticommutators; the first one was studied by Bender and Bettencourt [2], and links the $n$-nested anticommutator of the operators $q$ and $H$ to a simpler anti-commutator of $q$ and a polynomial version of $H$. This result was not proved, but only inferred by these authors. Only recently, a proof was provided in [5], based on the integral representation of operators, more precisely the generalized Weyl transform. Our approach consists in first transforming Bender and Bettencourt's identity into a simpler form, and then in providing two different proofs, one algebraic and another based on the properties of some operators. A third, purely analytic proof, is obtained by realizing the considered operators and looking at their action on a sufficiently large set of functions.

The second identity studied in this paper was introduced recently by Pain [3]: it expresses the commutator of monomials in operators $p$ and $q$ as a linear combination of their anti-commutators. We show that the use of generating functions not only gives a simple proof of this result, but also allows to derive the converse identity, expressing the anti-commutator of monomials of $p$ and $q$ as a linear combination of their commutators. In both cases, the Euler and Bernoulli polynomials play an important role.

The third identity appears in the context of non-Hermitian Hamiltonian systems, as studied by Figuieira de Morisson and Fring in [9]: the real and imaginary part of the non-Hermitian Hamiltonian can be shown, under assumptions that will be detailed later, to be related by a linear identity involving Euler numbers.

One of the main intents of this paper is to show that for a given identity on non-commutating operators, a variety of different proofs - each pertaining to a different area of mathematics - can be exhibited. On the way of each of these proofs, some interesting results, of either combinatorial, algebraic or analytical nature, may appear. 


\section{AN IDENTITY BY BENDER AND BETTENCOURT}

\section{A. Introduction}

In [2], C. Bender and L. Bettencourt inspect the first values of the operator

$$
\frac{1}{2^{n}}\{q, H\}_{n} ;
$$

for $n=0,1,2,3,4,5$ and 6 , they find respectively

$$
\begin{gathered}
1, \frac{1}{2}\{q, H\}, \frac{1}{2}\left\{q, H^{2}-\frac{1}{4}\right\}, \frac{1}{2}\left\{q, H^{3}-\frac{3}{4} H\right\} \\
\frac{1}{2}\left\{q, H^{4}-\frac{3}{2} H^{2}+\frac{5}{16}\right\}, \frac{1}{2}\left\{q, H^{5}-\frac{5}{2} H^{3}+\frac{25}{16} H\right\}
\end{gathered}
$$

and

$$
\frac{1}{2}\left\{q, H^{6}-\frac{15}{4} H^{4}+\frac{75}{16} H^{2}-\frac{61}{64}\right\} .
$$

This suggests the following result

$$
\frac{1}{2^{n}}\{q, H\}_{n}=\frac{1}{2}\left\{q, E_{n}\left(H+\frac{1}{2}\right)\right\}
$$

where, by a real tour de force, the authors identify $E_{n}(x)$ as the Euler polynomial of degree $n$, defined by the exponential generating function

$$
\sum_{n \geq 0} \frac{E_{n}(x)}{n !} z^{n}=\frac{2 e^{z x}}{e^{z}+1},|z|<2 \pi .
$$

Since this identity is only inferred in [2], we propose to prove it in several ways using algebraic or analytic methods.

\section{B. Preprocessing}

As a first step of the proof, we transform the identity (II.1) into the equivalent form

$$
\frac{1}{2^{n}}\left\{q, H-\frac{1}{2}\right\}_{n}=\frac{1}{2}\left\{q, E_{n}(H)\right\} .
$$

Next, we consider the following lemma.

Lemma 1. A polynomial $P_{n}(x)$ satisfies

$$
\frac{1}{2} P_{n}(x)+\frac{1}{2} P_{n}(x+1)=x^{n}
$$

if and only if

$$
P_{n}(x)=E_{n}(x),
$$

the Euler polynomial of degree $n$. 
Proof. The fact that the Euler polynomials satisfy

$$
E_{n}(x)+E_{n}(x+1)=2 x^{n}, n \in \mathbb{N},
$$

can be deduced from the generating function (II.2). The fact that this is an equivalence can be checked as follows: starting from

$$
E_{n}(x)+E_{n}(x+1)=P_{n}(x)+P_{n}(x+1)
$$

which shows that $P_{n}(x)$ is of degree $n$, and writing

$$
P_{n}(x)=a_{n}^{(n)} x^{n}+\ldots
$$

then, taking $x \rightarrow+\infty$ shows that $a_{n}^{(n)}$ is also the leading term of $E_{n}(x)$. Then applying the same argument to the new polynomial

$$
P_{n}(x)-a_{n}^{(n)} x^{n}
$$

shows by induction on $n$ that both polynomials $P_{n}$ and $E_{n}$ coincide.

Another proof of this Lemma uses the integral representation of Euler polynomials that can be found as formula 24.7.6 in [8],

$$
E_{n}(z)=\int_{-\infty}^{+\infty}\left(\imath u-\frac{1}{2}+z\right)^{n} \operatorname{sech}(\pi u) d u
$$

Start expanding

$$
P_{n}(x)=\sum_{k=0}^{n} p_{k}^{(n)} x^{k}
$$

and rewrite (II.4) as

$$
\frac{1}{2} \sum_{k=0}^{n} p_{k}^{(n)} x^{k}+\frac{1}{2} \sum_{k=0}^{n} p_{k}^{(n)}(x+1)^{k}=x^{n}
$$

Substitute $x \rightarrow \imath u-\frac{1}{2}+x$ in both sides and integrate again the function $\operatorname{sech}(\pi u)$, giving

$$
\frac{1}{2} \sum_{k=0}^{n} p_{k}^{(n)} E_{k}(x)+\frac{1}{2} \sum_{k=0}^{n} p_{k}^{(n)} E_{k}(x+1)=E_{n}(x) .
$$

Using the property (II.5) of Euler polynomials, deduce

$$
\sum_{k=0}^{n} p_{k}^{(n)} x^{k}=P_{n}(x)=E_{n}(x)
$$

Let us introduce next the symbolic notation, with $a \in \mathbb{R}$,

$$
(\{q, H\}+a)_{n}=\sum_{k=0}^{n}\left(\begin{array}{l}
n \\
k
\end{array}\right) a^{n-k}\{q, H\}_{k}
$$

and the convention $\{q, H\}_{0}=q$.

We will need the following identity, the proof of which is elementary. 
Lemma 2. For $a \in \mathbb{R}$ and $n \in \mathbb{N}$,

$$
\left\{q, H+\frac{a}{2}\right\}_{n}=(\{q, H\}+a)_{n} .
$$

Proof. Take the exponential generating function on both sides. On the left-hand side

$$
\sum_{n \geq 0}\left\{q, H+\frac{a}{2}\right\}_{n} \frac{z^{n}}{n !}=e^{z\left(H+\frac{a}{2}\right)} q e^{z\left(H+\frac{a}{2}\right)}
$$

while on the right-hand side

$$
\sum_{n \geq 0}(\{q, H\}+a)_{n} \frac{z^{n}}{n !}=e^{z a} e^{z H} q e^{z H}
$$

so that both generating functions coincide. Since they are analytic functions, the corresponding sequences coincide.

Using both previous lemmas, we can now deduce the following equivalence.

Proposition 3. An equivalent form of identity (II.1) (or (II.3)) is

$$
\frac{1}{2^{n}}\left\{q, H-\frac{1}{2}\right\}_{n}+\frac{1}{2^{n}}\left\{q, H+\frac{1}{2}\right\}_{n}=\left\{q, H^{n}\right\} .
$$

Proof. Assume first that

$$
\frac{1}{2^{n}}\left\{q, H-\frac{1}{2}\right\}_{n}=\frac{1}{2}\left\{q, E_{n}(H)\right\}
$$

holds. Then

$$
\frac{1}{2^{n}}\left\{q, H-\frac{1}{2}\right\}_{n}+\frac{1}{2^{n}}\left\{q, H+\frac{1}{2}\right\}_{n}=\left\{q, \frac{1}{2}\left(E_{n}(H)+E_{n}(H+1)\right)\right\}=\left\{q, H^{n}\right\}
$$

where we have used the property (II.5) of Euler polynomials.

Reciprocally, assume that

$$
\frac{1}{2^{n}}\left\{q, H-\frac{1}{2}\right\}_{n}+\frac{1}{2^{n}}\left\{q, H+\frac{1}{2}\right\}_{n}=\left\{q, H^{n}\right\}
$$

holds. As can be checked from their generating function (II.2), the Euler polynomials are Appell polynomials, i.e. they satisfy

$$
E_{n}(x)=\sum_{k=0}^{n}\left(\begin{array}{l}
n \\
k
\end{array}\right) E_{n-k}(0) x^{k}
$$

hence we have

$$
\left\{q, E_{n}(H)\right\}=\left\{q, \sum_{k=0}^{n}\left(\begin{array}{l}
n \\
k
\end{array}\right) E_{n-k}(0) H^{k}\right\}
$$

so that we need to compute

$$
\sum_{k=0}^{n}\left(\begin{array}{l}
n \\
k
\end{array}\right) E_{n-k}(0) \frac{1}{2^{k}}\left\{q, H-\frac{1}{2}\right\}_{k} \text { and } \sum_{k=0}^{n}\left(\begin{array}{l}
n \\
k
\end{array}\right) E_{n-k}(0) \frac{1}{2^{k}}\left\{q, H+\frac{1}{2}\right\}_{k}
$$


and to show that their sum is equal to

$$
\frac{1}{2^{n-1}}\left\{q, H-\frac{1}{2}\right\}_{n}
$$

By Lemma (2), the first sum is

$$
\sum_{k=0}^{n}\left(\begin{array}{l}
n \\
k
\end{array}\right) E_{n-k}(0) \frac{1}{2^{k}}\left\{q, H-\frac{1}{2}\right\}_{k}=\sum_{k=0}^{n}\left(\begin{array}{l}
n \\
k
\end{array}\right) E_{n-k}(0) \frac{1}{2^{k}}(\{q, H\}-1)_{k} .
$$

Applying (II.7), this is

$$
\begin{aligned}
\sum_{k=0}^{n}\left(\begin{array}{l}
n \\
k
\end{array}\right) E_{n-k}(0) \frac{1}{2^{k}} \sum_{l=0}^{k}\left(\begin{array}{c}
k \\
l
\end{array}\right)(-1)^{k-l}\{q, H\}_{l} & =\sum_{l=0}^{n}\left(\begin{array}{l}
n \\
l
\end{array}\right)\{q, H\}_{l} \sum_{p=0}^{n-l}\left(\begin{array}{c}
n-l \\
p
\end{array}\right) E_{n-l-p}(0) \frac{(-1)^{p}}{2^{p+l}} \\
& =\sum_{l=0}^{n}\left(\begin{array}{l}
n \\
l
\end{array}\right) \frac{\{q, H\}_{l}}{2^{l}} E_{n-k}\left(-\frac{1}{2}\right) .
\end{aligned}
$$

Thus

$$
\sum_{k=0}^{n}\left(\begin{array}{l}
n \\
k
\end{array}\right) E_{n-k}(0) \frac{1}{2^{k}}\left\{q, H-\frac{1}{2}\right\}_{k}=\sum_{l=0}^{n}\left(\begin{array}{l}
n \\
l
\end{array}\right) \frac{\{q, H\}_{l}}{2^{l}} E_{n-l}\left(-\frac{1}{2}\right) .
$$

and similarly

$$
\sum_{k=0}^{n}\left(\begin{array}{l}
n \\
k
\end{array}\right) E_{n-k}(0) \frac{1}{2^{k}}\left\{q, H+\frac{1}{2}\right\}_{k}=\sum_{l=0}^{n}\left(\begin{array}{l}
n \\
l
\end{array}\right) \frac{\{q, H\}_{l}}{2^{l}} E_{n-l}\left(\frac{1}{2}\right)
$$

so that the sum equals

$$
\frac{1}{2^{n}} \sum_{l=0}^{n}\left(\begin{array}{l}
n \\
l
\end{array}\right) \frac{\{q, H\}_{l}}{2^{l}}\left(E_{n-l}\left(-\frac{1}{2}\right)+E_{n-l}\left(\frac{1}{2}\right)\right)=\frac{1}{2^{n-1}} \sum_{l=0}^{n}\left(\begin{array}{l}
n \\
l
\end{array}\right) \frac{\{q, H\}_{l}}{2^{l}}\left(-\frac{1}{2}\right)^{n-l}
$$

where we have used (II.5) again.

The last sum is now identified by Lemma (2) as

$$
\frac{2}{2^{n}}(\{q, H\}-1)_{n}=\frac{1}{2^{n-1}}\left\{q, H-\frac{1}{2}\right\}_{n},
$$

and this is the desired result.

We conclude that proving (II.1) is equivalent to be proving the following result.

Theorem 4. If $p$ and $q$ are two operators that satisfy (I.1) and $H$ is the associated Hamiltonian defined as in (I.2), then for any integer $n \geq 0$,

$$
\frac{1}{2^{n}}(\{q, H\}-1)_{n}+\frac{1}{2^{n}}(\{q, H\}+1)_{n}=\left\{q, H^{n}\right\}
$$

with the symbolic notation $(\{q, H\}+a)_{n}$ as defined in (II.7).

Example 5. The case $n=1$ reads

$$
\frac{1}{2}(\{q, H\}-1)_{1}+\frac{1}{2}(\{q, H\}+1)_{1}=\{q, H\} .
$$

This is easily checked by expanding the left-hand side as

$$
\frac{1}{2}(\{q, H\}-1)_{1}+\frac{1}{2}(\{q, H\}+1)_{1}=\frac{1}{2}(\{q, H\}-1)+\frac{1}{2}(\{q, H\}+1)=\{q, H\} .
$$


Example 6. The case $n=2$ reads

$$
\frac{1}{4}(\{q, H\}-1)_{2}+\frac{1}{4}(\{q, H\}+1)_{2}=\left\{q, H^{2}\right\} .
$$

The left-hand side is now

$$
\frac{1}{4}(\{q, H\}-1)_{2}+\frac{1}{4}(\{q, H\}+1)_{2}=\frac{1}{2}\{q, H\}_{2}+\frac{1}{2}\{q, H\}_{0}=\frac{1}{2}\left(\{q, H\}_{2}+q\right) .
$$

But since $[q, H]_{2}=[[q, H], H]$ and $[q, H]=\imath p$, we deduce $[q, H]_{2}=[\imath p, H]=\imath[p, H]$. It then follows from $[p, H]=-\imath q$ that $[q, H]_{2}=q$.

We deduce

$$
\begin{aligned}
\frac{1}{2}\left(\{q, H\}_{2}+q\right) & =\frac{1}{2}\left(\{q, H\}_{2}+[q, H]_{2}\right) \\
& =\frac{1}{2}\left(q H^{2}+2 H q H+H^{2} q\right)+\frac{1}{2}\left(q H^{2}-2 H q H+H^{2} q\right) \\
& =q H^{2}+H^{2} q=\left\{q, H^{2}\right\}
\end{aligned}
$$

which is the desired result.

\section{An algebraic proof}

The first proof of Thm 4 (and thus of Bender's identity (II.1) is given in the case $n$ even only, replacing $n$ by $2 n$ in (II.8): its extension to the odd case follows the same lines. It starts with the expansion

$$
\frac{1}{2}(\{q, H\}-1)_{2 n}+\frac{1}{2}(\{q, H\}+1)_{2 n}=\sum_{k=0}^{n}\left(\begin{array}{l}
2 n \\
2 k
\end{array}\right)\{q, H\}_{2 k}
$$

in which $\{q, H\}_{2 k}$ is replaced by $\left\{[q, H]_{2 n-2 k}, H\right\}_{2 k}$ since $[q, H]_{2 n-2 k}=q$. We obtain

$$
\begin{array}{r}
\sum_{k=0}^{n}\left(\begin{array}{c}
2 n \\
2 k
\end{array}\right)\left\{[q, H]_{2 n-2 k}, H\right\}_{2 k}=\sum_{k=0}^{n}\left(\begin{array}{c}
2 n \\
2 k
\end{array}\right) \sum_{l=0}^{2 k}\left(\begin{array}{c}
2 k \\
l
\end{array}\right) H^{l}[q, H]_{2 n-2 k} H^{2 k-l} \\
=\sum_{k=0}^{n}\left(\begin{array}{c}
2 n \\
2 k
\end{array}\right) \\
\sum_{l=0}^{2 k}\left(\begin{array}{c}
2 k \\
l
\end{array}\right) H^{l}\left(\sum_{r=0}^{2 n-2 k}\left(\begin{array}{c}
2 n-2 k \\
r
\end{array}\right)(-1)^{r} H^{r} q H^{2 n-2 k-r}\right) H^{2 k-l} \\
=\sum_{k=0}^{n} \sum_{l=0}^{2 k} \sum_{r=0}^{2 n-2 k}\left(\begin{array}{c}
2 n \\
2 k
\end{array}\right)\left(\begin{array}{c}
2 k \\
l
\end{array}\right)\left(\begin{array}{c}
2 n-2 k \\
r
\end{array}\right)(-1)^{r} H^{l+r} q H^{2 n-r-l} \\
=\sum_{k=0}^{n} \sum_{l=0}^{2 k} \sum_{s=r+l=0}^{2 n}\left(\begin{array}{c}
2 n \\
2 k
\end{array}\right)\left(\begin{array}{c}
2 k \\
l
\end{array}\right)\left(\begin{array}{c}
2 n-2 k \\
s-l
\end{array}\right)(-1)^{s-l} H^{s} q H^{2 n-s} .
\end{array}
$$

We thus need to show that

$$
b_{s}=\sum_{k=0}^{n} \sum_{l=0}^{2 k}\left(\begin{array}{l}
2 n \\
2 k
\end{array}\right)\left(\begin{array}{c}
2 k \\
l
\end{array}\right)\left(\begin{array}{c}
2 n-2 k \\
s-l
\end{array}\right)(-1)^{s-l}= \begin{cases}0 & 1 \leq s \leq 2 n-1 \\
2^{2 n-1} & s=0, s=2 n\end{cases}
$$

Differentiating the identity

$$
\sum_{k=0}^{n}\left(\begin{array}{l}
2 n \\
2 k
\end{array}\right) x^{2 k} y^{2 n-2 k}=\frac{1}{2}(x+y)^{2 n}+\frac{1}{2}(x-y)^{2 n}
$$


$i$ times with respect to $x$ and $j$ times with respect to $y$, and then evaluating at $x=y=1$ gives

$$
\sum_{k=0}^{n}\left(\begin{array}{c}
2 n \\
2 k
\end{array}\right)\left(\begin{array}{c}
2 k \\
i
\end{array}\right)\left(\begin{array}{c}
2 n-2 k \\
j
\end{array}\right)=\left(\begin{array}{c}
2 n \\
i
\end{array}\right)\left(\begin{array}{c}
2 n-i \\
j
\end{array}\right) 2^{2 n-i-j-1}
$$

if $0 \leq i \leq n, 0 \leq j \leq n$ and $i+j \leq 2 n-1$, while

$$
\sum_{k=0}^{n}\left(\begin{array}{c}
2 n \\
2 k
\end{array}\right)\left(\begin{array}{c}
2 k \\
n
\end{array}\right)\left(\begin{array}{c}
2 n-2 k \\
n
\end{array}\right)=\frac{1}{2}\left(\begin{array}{c}
2 n \\
n
\end{array}\right)\left(1+(-1)^{n}\right)
$$

if $i=j=n$. We deduce, for $1 \leq s \leq 2 n-1$,

$$
\begin{aligned}
b_{s} & =\sum_{l=0}^{s} \sum_{k=0}^{n}\left(\begin{array}{c}
2 n \\
2 k
\end{array}\right)\left(\begin{array}{c}
2 k \\
l
\end{array}\right)\left(\begin{array}{c}
2 n-2 k \\
s-l
\end{array}\right)(-1)^{s-l}=\sum_{l=0}^{s}(-1)^{s-l}\left(\begin{array}{c}
2 n \\
l
\end{array}\right)\left(\begin{array}{c}
2 n-l \\
s-l
\end{array}\right) 2^{2 n-s-1} \\
& =2^{2 n-s-1}\left(\begin{array}{c}
2 n \\
s
\end{array}\right) \sum_{l=0}^{s}(-1)^{s-l}\left(\begin{array}{l}
s \\
l
\end{array}\right)=0 .
\end{aligned}
$$

A straightforward computation gives $b_{0}=b_{2 n}=2^{2 n-1}$. This concludes the proof.

\section{An operator-based proof}

Another version of the proof of Thm 4 is obtained now by identifying the nested commutators and anti-commutators $[q, H]_{k}$ and $\{q, H\}_{k}$ as powers of simple operators.

Define the operators $A_{H}$ and $B_{H}$ acting on $q$ as

$$
A_{H} q=[q, H], \quad B_{H} q=\{q, H\} .
$$

We first verify the following identities.

Lemma 7. The operators $A_{H}$ and $B_{H}$ satisfy, for $k \in \mathbb{N}$,

$$
A_{H}^{k} q=[q, H]_{k}, B_{H}^{k} q=\{q, H\}_{k}
$$

and

$$
\left(A_{H}+B_{H}\right)^{k} q=2^{k} q H^{k},\left(A_{H}-B_{H}\right)^{k} q=2^{k} H^{k} q .
$$

Moreover, $A_{H}$ and $B_{H}$ commute.

Proof. The identities (II.9) and (II.10) are easily proved by induction on $k$. Moreover, $B_{H} A_{H} q=$ $q H^{2}-H^{2} q=A_{H} B_{H} q$ so that $A_{H}$ and $B_{H}$ commute.

We obtain the final identity using (II.9) and (I.10) as follows:

$$
\begin{aligned}
\sum_{k=0}^{n}\left(\begin{array}{c}
2 n \\
2 k
\end{array}\right)\left\{[q, H]_{2 n-2 k}, H\right\}_{2 k} & =\sum_{k=0}^{n}\left(\begin{array}{c}
2 n \\
2 k
\end{array}\right) B_{H}^{2 k} A_{H}^{2 n-2 k} q \\
& =\frac{1}{2}\left(\left(A_{H}+B_{H}\right)^{2 n}+\left(A_{H}-B_{H}\right)^{2 n}\right) q \\
& =2^{2 n-1}\left(q H^{2 n}+H^{2 n} q\right)=2^{2 n-1}\left\{q, H^{2 n}\right\} .
\end{aligned}
$$




\section{E. Another Proof: an analytic approach}

The third proof of Thm 4 is purely analytic: we choose the realization

$$
p=x, q=\imath \frac{d}{d x} .
$$

This is a faithful realization [1]: any result proved with this realization holds for any other operators $p$ and $q$ that satisfy the commutation relation (I.1).

Next we consider the Hermite functions defined by the Rodrigues formula

$$
\psi_{n}(x)=(-1)^{n} \frac{1}{\sqrt{2^{n} n ! \sqrt{\pi}}} e^{\frac{x^{2}}{2}} \frac{d^{n}}{d x^{n}} e^{-x^{2}}, n \in \mathbb{N} .
$$

These functions satisfy the differential equation

$$
-\psi{ }_{n}(x)+x^{2} \psi_{n}(x)=(2 n+1) \psi_{n}(x)
$$

or equivalently

$$
H \psi_{n}(x)=\left(n+\frac{1}{2}\right) \psi_{n}(x),
$$

so that they are eigenfunctions of the Hamiltonian. Since they form a basis of $L^{2}(\mathbb{R})$, it is sufficient to verify (II.8) on this set of functions. But it is easily verified that

$$
\{q, H\}_{n} \psi_{l}(x)=\sum_{k=0}^{n}\left(\begin{array}{l}
n \\
k
\end{array}\right) H^{k} q H^{n-k} \psi_{l}(x)
$$

with, from (II.11),

$$
H^{n-k} \psi_{l}(x)=\left(l+\frac{1}{2}\right)^{n-k} \psi_{l}(x)
$$

and with

$$
q \psi_{l}(x)=\imath\left(\sqrt{\frac{l}{2}} \psi_{l-1}(x)-\sqrt{\frac{l+1}{2}} \psi_{l+1}(x)\right)
$$

so that

$$
\begin{gathered}
\{q, H\}_{n} \psi_{l}(x)=\sum_{k=0}^{n}\left(\begin{array}{l}
n \\
k
\end{array}\right) H^{k} q H^{n-k} \psi_{l}(x) \\
=\sum_{k=0}^{n}\left(\begin{array}{l}
n \\
k
\end{array}\right)\left(l-\frac{1}{2}\right)^{k} \imath \sqrt{\frac{l}{2}}\left(l+\frac{1}{2}\right)^{n-k} \psi_{l-1}(x)-\sum_{k=0}^{n}\left(\begin{array}{l}
n \\
k
\end{array}\right)\left(l+\frac{3}{2}\right)^{k} \imath \sqrt{\frac{l+1}{2}}\left(l+\frac{1}{2}\right)^{n-k} \psi_{l+1}(x) \\
=\sqrt[\imath]{\frac{l}{2}}(2 l)^{n} \psi_{l-1}(x)-\imath \sqrt{\frac{l+1}{2}}(2 l+2)^{n} \psi_{l+1}(x)=\imath 2^{n-\frac{1}{2}}\left(l^{n+\frac{1}{2}} \psi_{l-1}(x)-(l+1)^{n+\frac{1}{2}} \psi_{l+1}(x)\right) .
\end{gathered}
$$

We deduce

$$
\begin{aligned}
(\{q, H\}+1)_{n} \psi_{l}(x) & =\sum_{k=0}^{n}\left(\begin{array}{l}
n \\
k
\end{array}\right)\{q, H\}_{k} \psi_{l}(x) \\
& =\sum_{k=0}^{n}\left(\begin{array}{l}
n \\
k
\end{array}\right) \imath 2^{k-\frac{1}{2}}\left(l^{k+\frac{1}{2}} \psi_{l-1}(x)-(l+1)^{k+\frac{1}{2}} \psi_{l+1}(x)\right) \\
& =\imath \sqrt{\frac{l}{2}}(1+2 l)^{n} \psi_{l-1}(x)-\imath \sqrt{\frac{l+1}{2}}(2 l+3)^{n} \psi_{l+1}(x)
\end{aligned}
$$


and accordingly

$$
(\{q, H\}-1)_{n} \psi_{l}(x)=\imath \sqrt{\frac{l}{2}}(2 l-1)^{n} \psi_{l-1}(x)-\imath \sqrt{\frac{l+1}{2}}(2 l+1)^{n} \psi_{l+1}(x)
$$

so that

$$
\begin{aligned}
{\left[(\{q, H\}+1)_{n}+(\{q, H\}-1)_{n}\right] \psi_{l}(x) } & =\imath \sqrt{\frac{l}{2}}\left[(2 l+1)^{n}+(2 l-1)^{n}\right] \psi_{l-1}(x) \\
& -\imath \sqrt{\frac{l+1}{2}}\left[(2 l+3)^{n}+(2 l+1)^{n}\right] \psi_{l+1}(x) .
\end{aligned}
$$

Now

$$
\begin{aligned}
\left\{q, H^{n}\right\} \psi_{l}(x) & =q H^{n} \psi_{l}(x)+H^{n} q \psi_{l}(x) \\
& =\left(l+\frac{1}{2}\right)^{n} \imath\left[\sqrt{\frac{l}{2}} \psi_{l-1}(x)-\sqrt{\frac{l+1}{2}} \psi_{l+1}(x)\right] \\
& +\imath \sqrt{\frac{l}{2}}\left(l-\frac{1}{2}\right)^{n} \psi_{l-1}(x)-\imath \sqrt{\frac{l+1}{2}}\left(l+\frac{3}{2}\right)^{n} \psi_{l+1}(x)
\end{aligned}
$$

which proves the result.

\section{IDENTITIES ON COMMUTATORS AND ANTI-COMMUTATORS OF MONOMIALS}

\section{A. Introduction}

In this second part, we change notations compared to the first part: in order to follow J.-C. Pain's notations, we assume now that the operators $p$ and $q$ satisfy the more general commutation relation

$$
[p, q]=p q-q p=c,
$$

with $c \in \mathbb{C} \backslash\{0\}$. Bender's results in the former section correspond to $c=-\imath$.

In the recent publication [3], J.-C. Pain derived the following identity between commutators and anti-commutators of monomials of the operators $p$ and $q$.

Theorem 8. [3, eq. (43)]If $p$ and $q$ are two operators such that

$$
[p, q]=p q-q p=c,
$$

then the commutators $\left[p^{n}, q^{m}\right]$ and anti-commutators $\left\{p^{n}, q^{m}\right\}$ of monomials are related by the convolution identity

$$
\left[\frac{p^{n}}{n !}, \frac{q^{m}}{m !}\right]=-\sum_{k=1}^{\min (m, n)} c^{k} \frac{E_{k}(0)}{k !}\left\{\frac{p^{n-k}}{(n-k) !}, \frac{q^{m-k}}{(m-k) !}\right\}
$$

where $E_{k}(0)$ is the Euler polynomial /? J of degree $k$ evaluated at 0 , as defined by (II.2).

The proof given in [3] relies on the fact that if the identity

$$
\left[\frac{p^{n}}{n !}, \frac{q^{m}}{m !}\right]=\sum_{k=1}^{\min (m, n)} c^{k} \frac{v_{k}}{k !}\left\{\frac{p^{n-k}}{(n-k) !}, \frac{q^{m-k}}{(m-k) !}\right\}
$$

holds for some sequence $\left\{v_{k}\right\}$, then these numbers $v_{k}$ should satisfy (see [3])

$$
v_{k}+\sum_{l=1}^{k}\left(\begin{array}{l}
k \\
l
\end{array}\right) v_{l}=1
$$

which, with $v_{0}=1$, characterizes exactly these numbers as $v_{k}=-E_{k}(0), k \geq 1$. 


\section{B. A proof using generating functions}

We give here another proof based on generating functions: consider the bivariate exponential generating function of the left-hand side of (III.2)

$$
\sum_{m, n \geq 0}\left[p^{n}, q^{m}\right] \frac{u^{n}}{n !} \frac{v^{m}}{m !}=\left[e^{u p}, e^{v q}\right] .
$$

For the right-hand side, we have the bivariate generating function

$$
\begin{gathered}
\sum_{m, n \geq 0} \sum_{k=1}^{\min (m, n)} c^{k} k ! v_{k}\left(\begin{array}{c}
n \\
k
\end{array}\right)\left(\begin{array}{c}
m \\
k
\end{array}\right)\left\{p^{n-k}, q^{m-k}\right\} \frac{u^{n}}{n !} \frac{v^{m}}{m !}=\sum_{m, n \geq 0} \sum_{k=1}^{\min (m, n)} \frac{(c u v)^{k}}{k !} v_{k}\left\{\frac{(u p)^{n-k}}{(n-k) !}, \frac{(v q)^{m-k}}{(m-k) !}\right\} \\
=\left(\sum_{k=1}^{+\infty} \frac{(c u v)^{k}}{k !} v_{k}\right) \sum_{m, n \geq 0}\left\{\frac{(u p)^{n}}{n !}, \frac{(v q)^{m}}{m !}\right\}=\left(\sum_{k=1}^{+\infty} \frac{(c u v)^{k}}{k !} v_{k}\right)\left\{e^{u p}, e^{v q}\right\}
\end{gathered}
$$

and we deduce, denoting $z=c u v$,

$$
\left[e^{u p}, e^{v q}\right]=\left(\sum_{k=1}^{+\infty} \frac{z^{k}}{k !} v_{k}\right)\left\{e^{u p}, e^{v q}\right\} .
$$

But since $[p, q]=c$, using McCoy's identity [4, Formula (14)]

$$
[f(p), g(q)]=-\sum_{k \geq 1} \frac{(-c)^{k}}{k !} f^{(k)}(p) g^{(k)}(q)
$$

with $f(p)=\exp (u p)$ and $f(q)=\exp (v q)$, we deduce

$$
\left[e^{u p}, e^{v q}\right]=\left(1-e^{-z}\right) e^{u p} e^{v q}
$$

and, since $-\left\{e^{u p}, e^{v q}\right\}=\left[e^{u p}, e^{v q}\right]-2 e^{u p} e^{v q}$, we deduce

$$
\left\{e^{u p}, e^{v q}\right\}=\left(1+e^{-z}\right) e^{u p} e^{v q} .
$$

In fact, these two identities can be obtained without using McCoy's identity (III.4) as a consequence of the celebrated Baker-Campbell-Hausdorff formula that tells us a more precise result, namely that

$$
e^{p} e^{q}=e^{p+q+\frac{1}{2}[p, q]}=e^{p+q+\frac{c}{2}}
$$

and

$$
e^{q} e^{p}=e^{q+p-\frac{c}{2}}
$$

from these two formulas, we deduce

$$
e^{p+q}=e^{-\frac{c}{2}} e^{p} e^{q}=e^{\frac{c}{2}} e^{q} e^{p}
$$

and then (III.5) and (III.6).

Hence the commutators and anti-commutators are related as

$$
\left[e^{u p}, e^{v q}\right]=\frac{1-e^{-z}}{1+e^{-z}}\left\{e^{u p}, e^{v q}\right\}=\frac{e^{z}-1}{e^{z}+1}\left\{e^{u p}, e^{v q}\right\} .
$$

Identifying with (III.3) gives

$$
\sum_{k=1}^{+\infty} \frac{z^{k}}{k !} v_{k}=\frac{e^{z}-1}{e^{z}+1}
$$


or

$$
\sum_{k=0}^{+\infty} \frac{z^{k}}{k !} v_{k}=1+\frac{e^{z}-1}{e^{z}+1}=\frac{2 e^{z}}{1+e^{z}}=\sum_{k=0}^{+\infty} \frac{E_{k}(1)}{k !} z^{k}
$$

so that $v_{k}=E_{k}(1)$. Using (II.5), we deduce, for all $k \geq 1$,

$$
v_{k}=E_{k}(1)=-E_{k}(0) \text {. }
$$

\section{The reciprocal identity}

One of the interesting features of this generating function method is that it allows us to invert the identity (III.1), giving an expression of the anti-commutators in terms of the commutators. First recall that the Bernoulli numbers are defined by the exponential generating function

$$
\sum_{n \geq 0} \frac{B_{n}}{n !} z^{n}=\frac{z}{e^{z}-1},|z|<2 \pi
$$

We can now prove the following result.

Theorem 9. If $p$ and $q$ satisfy $[p, q]=c$ then for $m, n \geq 0$,

$$
\left\{\frac{p^{n}}{n !}, \frac{q^{m}}{m !}\right\}=\frac{2}{c}\left[\frac{p^{n+1}}{(n+1) !}, \frac{q^{m+1}}{(m+1) !}\right]+2 \sum_{k \geq 1}^{\min (m, n)} \frac{c^{k}}{k !} \frac{B_{k+1}}{k+1}\left[\frac{p^{n-k}}{(n-k) !}, \frac{q^{m-k}}{(m-k) !}\right]
$$

where $B_{n}$ is the $n$-th Bernoulli number.

Proof. First transform the identity

$$
\left[e^{u p}, e^{v q}\right]=\frac{e^{z}-1}{e^{z}+1}\left\{e^{u p}, e^{v q}\right\}
$$

into the equivalent

$$
\begin{aligned}
\left\{e^{u p}, e^{v q}\right\} & =\frac{e^{c u v}+1}{e^{c u v}-1}\left[e^{u p}, e^{v q}\right]=\left(1+\frac{2}{e^{z}-1}\right)\left[e^{u p}, e^{v q}\right] \\
& =\left(1+\frac{2}{z} \frac{z}{e^{z}-1}\right)\left[e^{u p}, e^{v q}\right]=\left(1+\frac{2}{z} \sum_{k \geq 0} \frac{z^{k}}{k !} B_{k}\right)\left[e^{u p}, e^{v q}\right] .
\end{aligned}
$$

Since $B_{0}=1$ and $B_{1}=-\frac{1}{2}$, this gives

$$
\left\{e^{u p}, e^{v q}\right\}=\left(1+\frac{2}{z}\left(1-\frac{1}{2} z+\sum_{k \geq 2} \frac{z^{k}}{k !} B_{k}\right)\right)\left[e^{u p}, e^{v q}\right]
$$

which simplifies to

$$
\left\{e^{u p}, e^{v q}\right\}=2\left(\frac{1}{z}+\sum_{k \geq 1} \frac{z^{k}}{k !} \frac{B_{k+1}}{k+1}\right)\left[e^{u p}, e^{v q}\right]
$$

Expanding the exponentials in the commutator and anti-commutator and identifying the corresponding powers of $u$ and $v$ with $z=c u v$ gives the desired result. 
As remarked by J.-C. Pain in [3], using the fact that

$$
E_{k}(0)=-2\left(2^{k+1}-1\right) \frac{B_{k+1}}{k+1}
$$

the original identity (III.1) can be rewritten as

$$
\left[\frac{p^{n}}{n !}, \frac{q^{m}}{m !}\right]=2 \sum_{k=1}^{\min (m, n)} \frac{c^{k}}{k !}\left(2^{k+1}-1\right) \frac{B_{k+1}}{k+1}\left\{\frac{p^{n-k}}{(n-k) !}, \frac{q^{m-k}}{(m-k) !}\right\}
$$

which now shows more resemblance with its reciprocal version (III.8).

\section{An analytic approach}

We look now for another proof of the identity (III.1) using an analytic approach: the operators $p$ and $q$ are realized as

$$
p=c f^{\prime}(x), q f(x)=x f(x)
$$

so that they satisfy the condition

$$
[p, q]=c,
$$

and we look at the action of the commutators $\left[p^{n}, q^{m}\right]$ and anti-commutators $\left\{p^{n}, q^{m}\right\}$ on the monomials $f(x)=x^{l}$ where we assume that $l \geq n$. An easy computation gives [? ]

$$
\left[\frac{p^{n}}{n !}, \frac{q^{m}}{m !}\right] x^{l}=\frac{c^{n}}{m !}\left(\left(\begin{array}{c}
m+l \\
n
\end{array}\right)-\left(\begin{array}{c}
l \\
n
\end{array}\right)\right) x^{l-n+m}
$$

and

$$
\left\{\frac{p^{n-k}}{(n-k) !}, \frac{q^{m-k}}{(m-k) !}\right\} x^{l}=\frac{c^{n-k}}{(m-k) !}\left(\left(\begin{array}{c}
m-k+l \\
n-k
\end{array}\right)+\left(\begin{array}{c}
l \\
n-k
\end{array}\right)\right) x^{l-n+m} .
$$

Hence we need to prove that

$$
\left(\begin{array}{c}
m+l \\
n
\end{array}\right)-\left(\begin{array}{l}
l \\
n
\end{array}\right)=-\sum_{k=1}^{\min (m, n)} E_{k}(0)\left(\begin{array}{c}
m \\
k
\end{array}\right)\left(\left(\begin{array}{c}
m-k+l \\
n-k
\end{array}\right)+\left(\begin{array}{c}
l \\
n-k
\end{array}\right)\right)
$$

or equivalently, since the Euler polynomials satisfy $E_{k}(1)=-E_{k}(0)$ for $k \geq 1$,

$$
\sum_{k=0}^{\min (m, n)} E_{k}(0)\left(\begin{array}{c}
m \\
k
\end{array}\right)\left(\begin{array}{c}
m-k+l \\
n-k
\end{array}\right)=\sum_{k=0}^{\min (m, n)} E_{k}(1)\left(\begin{array}{c}
m \\
k
\end{array}\right)\left(\begin{array}{c}
l \\
n-k
\end{array}\right) .
$$

We will prove the following more general result.

Theorem 10. The following identity holds

$$
\sum_{k=0}^{\min (m, n)} z^{k}\left(\begin{array}{c}
m \\
k
\end{array}\right)\left(\begin{array}{c}
m-k+l \\
n-k
\end{array}\right)=\sum_{k=0}^{\min (m, n)}(z+1)^{k}\left(\begin{array}{c}
m \\
k
\end{array}\right)\left(\begin{array}{c}
l \\
n-k
\end{array}\right)
$$

As a consequence, the Euler polynomials satisfy the identity

$$
\sum_{k=0}^{\min (m, n)} E_{k}(z)\left(\begin{array}{c}
m \\
k
\end{array}\right)\left(\begin{array}{c}
m-k+l \\
n-k
\end{array}\right)=\sum_{k=0}^{\min (m, n)} E_{k}(z+1)\left(\begin{array}{c}
m \\
k
\end{array}\right)\left(\begin{array}{c}
l \\
n-k
\end{array}\right) .
$$

The particular case $z=0$ gives the desired identity (III.9). 
Proof. We first proceed to prove the first identity (III.10). Defining

$$
P(z)=\sum_{k=0}^{\min (m, n)} z^{k}\left(\begin{array}{c}
m \\
k
\end{array}\right)\left(\begin{array}{c}
l \\
n-k
\end{array}\right), Q(z)=\sum_{k=0}^{\min (m, n)} z^{k}\left(\begin{array}{c}
m \\
k
\end{array}\right)\left(\begin{array}{c}
m-k+l \\
n-k
\end{array}\right),
$$

we thus need to show that $P(z+1)=Q(z)$. Expanding each $(z+1)^{k}$ in the expression for $P(z+1)$ gives

$$
\begin{aligned}
\sum_{k=0}^{\min (m, n)}(z+1)^{k}\left(\begin{array}{c}
m \\
k
\end{array}\right)\left(\begin{array}{c}
l \\
n-k
\end{array}\right) & =\sum_{k=0}^{\min (m, n)} \sum_{j=0}^{k}\left(\begin{array}{l}
k \\
j
\end{array}\right) z^{j}\left(\begin{array}{c}
m \\
k
\end{array}\right)\left(\begin{array}{c}
l \\
n-k
\end{array}\right) \\
& =\sum_{j=0}^{\min (m, n)}\left(\begin{array}{c}
m \\
j
\end{array}\right) \sum_{k=0}^{m-j}\left(\begin{array}{c}
m-j \\
k
\end{array}\right)\left(\begin{array}{c}
l \\
n-k-j
\end{array}\right) z^{j}
\end{aligned}
$$

Considering the coefficient of $z^{j}$ in this last expression, we see that the identity to be proved is

$$
\left(\begin{array}{c}
m-j+l \\
n-j
\end{array}\right)=\sum_{k=0}^{m-j}\left(\begin{array}{c}
m-j \\
k
\end{array}\right)\left(\begin{array}{c}
l \\
n-k-j
\end{array}\right) .
$$

This is known as the Chu-Vandermonde identity, and is a direct consequence of the identity

$$
(1+t)^{m-j+l}=(1+t)^{m-j}(1+t)^{l}
$$

by considering the coefficient of $t^{n-j}$ in each side.

Another proof based on hypergeometric functions is as follows: identify each of the polynomials $P$ and $Q$ as a Gauß hypergeometric function

$$
P(z)=\left(\begin{array}{l}
l \\
n
\end{array}\right){ }_{2} F_{1}\left(\begin{array}{c}
-m,-n \\
l+1-n
\end{array} ; z\right), Q(z)=\left(\begin{array}{c}
m+l \\
n
\end{array}\right){ }_{2} F_{1}\left(\begin{array}{c}
-m,-n \\
-l-m
\end{array} ;-z\right) .
$$

The desired identity $P(z+1)=Q(z)$ is a simple consequence of the linear transformation formula for the Gauß hypergeometric function that appears in [6] as identity 15.3.6,

$$
\begin{aligned}
{ }_{2} F_{1}\left(\begin{array}{c}
a, b \\
c
\end{array} ; z\right) & =\frac{\Gamma(c) \Gamma(c-a-b)}{\Gamma(c-a) \Gamma(c-b)}{ }_{2} F_{1}\left(\begin{array}{c}
a, b \\
a+b-c+1
\end{array} ; 1-z\right) \\
& +(1-z)^{c-a-b} \frac{\Gamma(c) \Gamma(a+b-c)}{\Gamma(a) \Gamma(b)}{ }_{2} F_{1}\left(\begin{array}{c}
c-a, c-b \\
c-a-b+1
\end{array} ; 1-z\right)
\end{aligned}
$$

with the choice $a=-m, b=-n, c=l+1-n$; note that the second term in the right-hand side vanishes since we assumed $-n+l+1>0$ and since $\Gamma(-n)=+\infty$.

The last step of the proof is to transform the general identity (III.10) into identity (III.11) for Euler polynomials; this is obtained by using the integral representation (II.6) of Euler polynomials. Replacing $z^{k}$ by $\left(\imath u-\frac{1}{2}+z\right)^{k}$ on each side of (III.10) and integrating over $u$ against the $\operatorname{sech}(\pi u)$ function gives the desired result.

\section{E. A generalization}

Exploiting the linearity of identities (III.1) and (III.8) allows to extend them to more general functions. We explicit this generalization in the case of two functions $f(p)$ and $g(p)$ that we assume regular enough to have an integral representation as Fourier transforms:

$$
f(p)=\int_{\mathbb{R}} \tilde{f}(u) e^{\imath u p} d u, g(q)=\int_{\mathbb{R}} \tilde{g}(v) e^{\imath v q} d q .
$$


Theorem 11. Assume that the functions $f$ and $g$ have the Fourier integral representation (III.12) for the operators $p$ and $q$ such that $[p, q]=c$. Denote moreover

$$
F(p)=\int_{\mathbb{R}} \frac{\tilde{f}(u)}{\imath u} e^{\imath u p} d u
$$

an antiderivative of $f$ and accordingly $G(p)$ for an antiderivative of $g$.

Then we have

$$
[f(p), g(q)]=-\sum_{k \geq 1} E_{k}(0) \frac{c^{k}}{k !}\left\{f^{(k)}(p), g^{(k)}(q)\right\}
$$

and

$$
\{f(p), g(q)\}=\frac{2}{c}[F(p), G(q)]+2 \sum_{k \geq 1} \frac{B_{k+1}}{k+1} \frac{c^{k}}{k !}\left[f^{(k)}(p), g^{(k)}(q)\right] .
$$

Additionally to McCoy's identity [4, Formula (14)]

$$
[f(p), g(q)]=-\sum_{k \geq 1} \frac{(-c)^{k}}{k !} f^{(k)}(p) g^{(k)}(q),
$$

we have the obvious

$$
\{f(p), g(q)\}=2 f(p) g(q)+\sum_{k \geq 1} \frac{(-c)^{k}}{k !} f^{(k)}(p) g^{(k)}(q)
$$

and the two last cases are

$$
f(p) g(q)=\frac{1}{c}[F(p), G(q)]-\sum_{k \geq 0} \frac{B_{k+1}}{k+1} \frac{(-c)^{k}}{k !}\left[f^{(k)}(p), g^{(k)}(q)\right]
$$

and

$$
f(p) g(q)=\frac{1}{2} \sum_{k \geq 0} \frac{E_{k}(0)}{k !}(-c)^{k}\left\{f^{(k)}(p), g^{(k)}(q)\right\} .
$$

Proof. We give the proof of the first identity only, the other proofs follow the same pattern.

The commutator $[f(p), g(q)]$ is computed from the integral representations (III.12) as

$$
\iint_{\mathbb{R}^{2}} \tilde{f}(u) \tilde{g}(v)\left[e^{\imath u p}, e^{\imath v q}\right] d u d v=\iint_{\mathbb{R}^{2}} \tilde{f}(u) \tilde{g}(v) \frac{e^{-z}-1}{e^{-z}+1}\left\{e^{\imath u p}, e^{\imath v q}\right\} d u d v
$$

where we have used (III.7) replacing $(u, v)$ by $(\imath u, \imath v)$ and remarking that $z=c u v$ is changed to $-z$. Now writing

$$
\frac{e^{-z}-1}{e^{-z}+1}=\frac{1-e^{z}}{1+e^{z}}=1-\frac{2 e^{z}}{e^{z}+1}=1-\sum_{k \geq 0} \frac{E_{k}(1)}{k !} z^{k}
$$

we deduce

$$
\begin{aligned}
\iint_{\mathbb{R}^{2}} \tilde{f}(u) \tilde{g}(v)\left[e^{\imath u p}, e^{\imath v q}\right] d u d v & =\iint_{\mathbb{R}^{2}} \tilde{f}(u) \tilde{g}(v)\left(1-\sum \frac{E_{k}(1)}{k !} z^{k}\right)\left\{e^{\imath u p}, e^{\imath v q}\right\} d u d v \\
& =\iint_{\mathbb{R}^{2}} \tilde{f}(u) \tilde{g}(v)\left\{e^{\imath u p}, e^{\imath v q}\right\} d u d v \\
& -\sum_{k \geq 0} \frac{E_{k}(1) c^{k}}{k !} \iint_{\mathbb{R}^{2}} u^{k} \tilde{f}(u) v^{k} \tilde{g}(v)\left\{e^{\imath u p}, e^{\imath v q}\right\} d u d v .
\end{aligned}
$$


The first right-hand side term is simply the anti-commutator $\{f(p), g(q)\}$. Each integral in the second term is identified as

$$
\int_{\mathbb{R}} u^{k} \tilde{f}(u) e^{\imath u p} d u=(-\imath)^{k} f^{(k)}(p),
$$

and the same for $g$. We deduce the second right-hand side term as

$$
\sum_{k \geq 0} \frac{E_{k}(1)(-c)^{k}}{k !}\left\{f^{(k)}(p), g^{(k)}(q)\right\} .
$$

Hence the right-hand side reads

$$
\{f(p), g(q)\}-\sum_{k \geq 0} \frac{E_{k}(1)(-c)^{k}}{k !}\left\{f^{(k)}(p), g^{(k)}(q)\right\}=-\sum_{k \geq 1} \frac{E_{k}(1)(-c)^{k}}{k !}\left\{f^{(k)}(p), g^{(k)}(q)\right\} .
$$

The final result is obtained by remarking that $E_{k}(1)=(-1)^{k} E_{k}(0), k \geq 0$.

\section{NON-HERMITIAN HAMILTONIAN SYSTEMS}

\section{A. Figuieira de Morisson and Fring's results}

Another context in which the Euler numbers appear naturally is the study of non-Hermitian Hamiltonian systems: in [9], Figuieira de Morisson and Fring consider a system ruled by an Hamiltonian $H$ that is not Hermitian but PT-symmetric, hence pseudo-Hermitian [11]; it is assumed that $H$ is similar to the Hermitian Hamiltonian $h$, and that the similarity transformation can be realized under the form

$$
h=e^{\frac{q}{2}} H e^{-\frac{q}{2}}
$$

for some Hermitian operator $q$. Writing the non-Hermitian Hamiltonian

$$
H=h_{0}+\imath h_{1}
$$

where $h_{0}^{\dagger}=h_{0}$ and $h_{1}^{\dagger}=h_{1}$, the pseudo-Hermitianity condition

$$
H^{\dagger}=e^{q} H e^{-q}
$$

is expressed as

$$
e^{q}\left(h_{0}-\imath h_{1}\right) e^{-q}=h_{0}+\imath h_{1}
$$

or equivalently as

$$
h_{0}-e^{q} h_{0} e^{-q}=\imath\left(h_{1}+e^{q} h_{1} e^{-q}\right) .
$$

This equation is solved in $h_{1}$ as a function of $h_{0}$ in [9], looking for coefficients $\kappa_{n}$ such that

$$
h_{1}=\imath \sum_{n=1} \frac{\kappa_{n}}{n !}\left[h_{0}, q\right]_{n} .
$$

In order to comply with the notations in [9], note that in this section, the nested commutator in (IV.3) is now iterated from the left:

$$
\left[h_{0}, q\right]_{n}=\left[q,\left[q, \ldots,\left[q, h_{0}\right]\right]\right]
$$


It is found in [9] that all even-index $\kappa_{2 n}$ numbers vanish and that

$$
\kappa_{1}=\frac{1}{2}, \kappa_{3}=-\frac{1}{4}, \kappa_{5}=\frac{1}{2}, \kappa_{7}=-\frac{17}{8}, \kappa_{9}=\frac{31}{2} \ldots
$$

(note the sign error on $\kappa_{9}$ in [9]). Then $h$ is computed using (IV.1) and it is found that

$$
h=\sum_{n \geq 0} \frac{\lambda_{n}}{2^{n} n !}\left[h_{0}, q\right]_{n}
$$

where the coefficients $\lambda_{n}$ are related to the $\kappa_{n}$ by

$$
\lambda_{n}=1-\sum_{m=0}^{n} 2^{m}\left(\begin{array}{c}
n \\
m
\end{array}\right) \kappa_{m} .
$$

These coefficients are identified as $\lambda_{2 n+1}=0$ and

$$
\lambda_{0}=1, \lambda_{2}=-1, \lambda_{4}=5, \lambda_{6}=-61 \ldots
$$

from where it is concluded (without proof) that

$$
\lambda_{2 n}=2^{n} E_{n}\left(\frac{1}{2}\right)
$$

\section{B. A proof and why Euler numbers appear here}

In order to prove these results, we'll use a new method based on symbolic computation in the spirit of the classical umbral calculus as introduced in [12], see also [13]. We define an Euler symbol $\mathcal{E}$ such that

$$
\mathcal{E}^{n}=E_{n}(0)
$$

the principle of umbral calculus is to perform the computations replacing the sequence of numbers $E_{n}$ by the powers $\mathcal{E}^{n}$ and to obtain the final result by the reverse substitution of each $\mathcal{E}^{n}$ by its counterpart $E_{n}$.

For example,

$$
e^{z \mathcal{E}}=\sum_{n \geq 0} \frac{\mathcal{E}^{n}}{n !} z^{n}=\sum_{n \geq 0} \frac{E_{n}(0)}{n !} z^{n}=\frac{2}{e^{z}+1}
$$

and more generally

$$
e^{z(\mathcal{E}+x)}=\sum_{n \geq 0} \frac{(\mathcal{E}+x)^{n}}{n !} z^{n}=\sum_{n \geq 0} \frac{E_{n}(x)}{n !} z^{n}=\frac{2 e^{z x}}{e^{z}+1}
$$

so that $\mathcal{E}+x$ is the symbol for the Euler polynomial, in the sense that

$$
(\mathcal{E}+x)^{n}=E_{n}(x) .
$$

The fundamental property of Euler symbols that explains their appearance in this context is the following extension of the identity (II.5).

Lemma 12. For any analytic function $f$,

$$
f(z+\mathcal{E})+f(z+\mathcal{E}+1)=2 f(z)
$$


Proof. By linearity, this result needs only be proved for monomials $f(z)=z^{n}$ : it then reduces to identity (II.5).

We are now in position to prove the following results.

Theorem 13. The equation (IV.2) has a solution

$$
h_{1}=\imath h_{0}-\imath \sum_{n \geq 0} \frac{E_{n}(0)}{n !}\left[q, h_{0}\right]_{n}
$$

The corresponding value of the Hamiltonian $h$ is

$$
h=\sum_{n \geq 0} \frac{E_{n}\left(\frac{1}{2}\right)}{n !}\left[q, h_{0}\right]_{n} .
$$

Hence the coefficients $\lambda_{n}$ in IV.4 and $\kappa_{n}$ in (IV.3) are respectively equal to

$$
\kappa_{n}= \begin{cases}0, & n=0 \\ -E_{n}(0), & n>0\end{cases}
$$

and

$$
\lambda_{n}=2^{n} E_{n}\left(\frac{1}{2}\right), n \geq 0 .
$$

Proof. Rewrite first the right-hand side of (IV.7) as

$$
h_{1}=\imath h_{0}-\imath e^{q \mathcal{E}} h_{0} e^{-q \mathcal{E}}
$$

deduce

$$
\begin{aligned}
h_{1}+e^{q} h_{1} e^{-q} & =\imath h_{0}-\imath e^{q \mathcal{E}} h_{0} e^{-q \mathcal{E}}+e^{q}\left(\imath h_{0}-\imath e^{q \mathcal{E}} h_{0} e^{-q \mathcal{E}}\right) e^{-q} \\
& =\imath\left(h_{0}+e^{q} h_{0} e^{-q}\right)-\imath\left(e^{q \mathcal{E}} h_{0} e^{-q \mathcal{E}}+e^{q(\mathcal{E}+1)} h_{0} e^{-q(\mathcal{E}+1)}\right) .
\end{aligned}
$$

Applying (IV.6) with $z=0$ and the function $f(\mathcal{E})=e^{q \mathcal{E}} h_{0} e^{-q \mathcal{E}}$ reduces the second term in the right-hand side to $2 h_{0}$ so that

$$
h_{1}+e^{q} h_{1} e^{-q}=\imath\left(-h_{0}+e^{q} h_{0} e^{-q}\right)
$$

and $h_{1}$ is a solution to (IV.2). The corresponding value of $h$ is

$$
\begin{aligned}
h & =e^{\frac{q}{2}}\left(h_{0}+\imath h_{1}\right) e^{-\frac{q}{2}}=e^{\frac{q}{2}}\left(h_{0}-h_{0}+e^{q \mathcal{E}} h_{0} e^{-q \mathcal{E}}\right) e^{-\frac{q}{2}} \\
& =e^{q\left(\mathcal{E}+\frac{1}{2}\right)} h_{0} e^{-q\left(\mathcal{E}+\frac{1}{2}\right)} .
\end{aligned}
$$

Expanding the exponentials in the formula gives the result (IV.8).

\section{CONCLUSION}

We have studied three identities on commutators and anti-commutators in which the Euler and Bernoulli numbers play a fundamental role. An open problem and desirable result at this point would be to link these identities with the combinatorial meaning of the Euler numbers: see [10] for a possible application.

[1] P. Blasiak and P. Flajolet. Combinatorial models of creation-annihilation, Séminaire Lotharingien de Combinatoire, 65.B65c, 1-78, 2011 
[2] C. M. Bender and Luis M.A. Bettencourt, Multiple-scale analysis of quantum systems, Phys. Rev. D, 54-12, 7710-7723, 1996

[3] J.-C. Pain, Commutation relations of operator monomials, J. Phys. A: Math. Theor. 46, 035304, 2013

[4] N. H. McCoy, On Commutation Formulas in the Algebra of Quantum Mechanic, Transactions of the American Mathematical Society, 3-4, 793-806, Oct. 1929

[5] H. B. Domingo and E. A. Galapon, Generalized Weyl transform for operator ordering: Polynomial functions in phase space, Journal of Mathematical Physics 56, 022104, 2015

[6] M. Abramowitz and I.A. Stegun, Handbook of mathematical functions: with formulas, graphs, and mathematical tables. New York: Dover Publications, 1970

[7] E. Elizalde, Ten physical applications of spectral zeta functions, Springer, 1995

[8] F. W. Olver, D. W. Lozier, R. F. Boisvert, and C. W. Clark, NIST Handbook of Mathematical Functions (1st ed.). Cambridge University Press, New York, NY, USA, 2010.

[9] C. Figuieira de Morisson and A. Fring, Time evolution of non-Hermitian Hamiltonian systems, J. Phys. A: Math. Gen. 39 9269, 2006

[10] A. Hodges and C.V. Sukumar, Bernoulli, Euler, permutations and quantum algebras, Proc. R. Soc. A, 463, 2401-2412, 2007

[11] A. Mostafazadeh, Pseudo-Hermiticity versus PT-Symmetry: the necessary condition for the reality of the spectrum of a non-Hermitian Hamiltonian, J. Math. Phys. 43, 205-214, 2002

[12] S. Roman, The umbral calculus, Academic Press, 1984

[13] I. M. Gessel, Applications of the classical umbral calculus, Algebra Universalis, Volume 49, Issue 4, pp 397-434, October 2003 